\title{
Complemento a la Bibliografía de y Sobre Juan Carlos Onetti
}

Hace algunos años Hugo J. Verani publicó una "Contribución a la bibliografía de Juan Carlos Onetti"' en Revista Iberoamericana (Pittsburgh, Vol. XXXVIII, No. 80 julioseptiembre de 1972, pp. 523-548) y la actualizó en la colección de artículos titulada Onetti, Jorge Ruffinelli, preparador (Montevideo: Biblioteca de Marcha, 1973, pp. 267291). La bibliografía aquí presentada completa y corrige la más reciente de Verani a la vez que agrega referencias nuevas, señaladas con *. Se sigue el mismo sistema de ordenación para facilitar el uso de esta nueva bibliografía como complemento. Se presentan íntegras las referencias a los cuentos, en las que se incluyen las colecciones donde se puede encontrar cada cuento, así como las de los artículos periodísticos escritos bajo los seudónimos de "Periquito el Aguador' ' y "Grucho Marx", Las otras partes sirven de suplemento, pero siempre se ofrece la referencia más completa.

Douglass College, Rutgers University

MARILYN FRANKENTHALER

\section{CUENTOS}

Abreviaturas de colecciones:

SR: Un sueño realizado y otros cuentos. Montevideo: Ediciones Número, 1951.

IT: El infierno tan temido. Montevideo: Ediciones Asir, 1962.

JSR: Jacob y el otro. Un sueño realizado y otros cuentos. Montevideo: Ediciones de la Banda Oriental, 1965.

C: Cuentos completos. Buenos Aires: Centro Editor de América Latina, S.A., 1967.

TN: Tres novelas. Montevideo: Editorial Alfa, 1967. 
NRO: La novia robada y otros cuentos. Buenos Aires: Centro Editor de América Latina, 1968.

CC: Cuentos completos. Caracas: Monte Avila Editores C.A., 1968.

OC: Obras completas. México: M. Aguilar, Editor, S.A.; 1970.

TA: Tiempo de abrazar y los cuentos de 1933 a 1950. Montevideo: Arca Editorial S.R.L., 1974.

"Avenida de Mayo-Diagonal-Avenida de Mayo." La Prensa (Buenos Aires), Año LXIV, No. 22.958 (1 de enero de 1933), 8 a sección, p. 4. Reproducido en Marcha (Montevideo), Año XI, No. 519 (17 de marzo de 1950), p. 14. (Colec.: TA).

“El obstáculo." La Nación (Buenos Aires), Año LXVI, No. 23.050 (6 de octubre de 1935), 2a sección, p. 3. (Colec.: TA.)

"El posible Baldi.", La Nación (Buenos Aires), Año LXVII, No. 23.398 (20 de septiembre de 1936), 5a sección, p. 2. (Colec.:_TA.)

"Convalescencia." Marcha (Montevideo), Año II, No. 34 (10 de febrero de 1940), Suplemento Literario (No. 4), pp. 3-5. Firmado: "H. C. Ramos". Reproducido en Latinoamérica (Buenos Aires), Año I, No. 1 (diciembre de 1972), pp. 51-56. (Colec.: TA.).

"Un sueño realizado." La Nación(Buenos Aires), Año LXXII, No. 25.143 (6 de julio de 1941), 2a sección, pp. 3-4. Reproducido en Marcha (Montevideo), Año X, No. 435 (2 de julio de 1948), pp. 14-15 y Año X, No. 436 (9 de julio de 1948), pp. 14-15.

(Colec.: SR, JSR, C, NRO, CC, OC y TA.)

"Mascarada." Apex(Montevideo), No. 2 (febrero de 1943), pp. 4-7. Reproducido en La Nacion (Buenos Aires), Año LXXIV, No. 25.778 (4 de abril de 1943), 2a sección, p. 2. También reproducido en Marcba (Montevideo), Año VI, No. 250 (15 de septiembre de 1944), pp. 14-15. (Colec.: IT, C, NRO, CC, OC y TA.)

"Bienvenido, Bob." La Nación (Buenos Aires), Año LXXV, No. 26.365 (12 de noviembre de 1944), 2 a sección, pp. 2 y 4. (Colec.: SR, JSR, C, NRO, CC, OC y . TA.)

“La larga historia." Alfar(Montevideo), Año XXII, No. 84 (1944), pp. 59-64. (Colec.: TA.) 
“Nueve de Julio." Marcha (Montevideo), Año VII, No. 314 (28 de diciembre de 1945), p. 14. (Presentado como fragmento de La cara de la desgracia pero no incluido. Coleccionado como cuento en $T A$.)

"Regreso al sur." La Nación (Buenos Aires), Año LXXVII, No. 26.894 (28 de abril de 1946), 2a sección, p. 2. (Colec.: TA.)

"Esbjerg, en la costa." La Nación (Buenos Aires), Año LXXVII, No. 27.095 (17 de noviembre de 1946), 2a sección, p. 2. (Colec.: SR, JSR, C, NRO, CC, OC y TA.)

"La casa en la arena." La Nación(Buenos Aires), Año LXXX, No. 27.922 (3 de abril de 1949), 2a sección, p. 4. (Colec.: SR, JSR, C, NRO, CC, OC y TA.)

"El álbum."' Sur (Buenos Aires), Nos. 219-220 (enero-febrero de 1953), pp. 66-79. (Colec.: IT, C, NRO, CC у OC.)

"Historia del Caballero de la Rosa y de la Virgen encinta que vino de Liliput." Entregas de la Licorne (Montevideo), No. 8 (1956), pp. 45-63. (Colec.: IT, C, NRO, CC y OC.)

“El infierno tan temido." Ficción (Buenos Aires), No. 5 (enero-febrero de 1957), pp. 6071. (Colec.: IT, C, NRO, CC y OC.)

“Jacob y el otro", en Ceremonia secreta y otros cuentos de América Latina. Garden City, New York: Ediciones Interamericanas, Doubleday and Company, Inc., 1961, pp. 349-389. (Colec.: $C, T N, C C$ y $O C$.)

"Justo el treintaiuno." Marcha (Montevideo), Año XXVI, No. 1220 (28 de agosto de 1964), 2a sección, pp. 23-24. (Colec.: C, CC y OC.)

"La novia robada." Papeles. Revista del Ateneo de Caracas (Caracas), No. 6 (abril-mayojunio de 1968), pp. 7-23. Reproducido en libro La novia robada. Buenos Aires: Siglo Veintiuno Editores, S.A., 1973. 68 pp. (Precede: Ricardo Piglia, "Onetti por Onetti," pp. 9-17.)(Colec.: NRO y OC.)

"Matias el telegrafista." Macedonio (Buenos Aires), No. 8 (1970), pp. 37-52. (Sin autorización del autor.) Publicado en Marcha (Montevideo), Año XXXIII, No. 1560 (10 de septiembre de 1971), pp. 30-31. Reproducido en La otra mitad del amor contado por ocho hombres. 2a ed. aumentada. Montevideo: Arca Editorial, 1972, pp. 81-97. 
" "Las mellizas", (Primera versión abreviada). Crisis (Buenos Aires), Año 1, No. 2 junio de 1973), pp. 32-35.

\section{NOVELAS}

* La muerte y la nina. Buenos Aires: Ediciones Corregidor, 1973. 135 pp.

\section{COLECCIONES DE CUENTOS Y NOVELAS}

Las máscaras del amor. Buenos Aires: Centro Editor de América Latina, 1968. 105 pp. Selección de Emir Rodríguez Monegal. (Presentación: Emir Rodríguez Monegal, p. 7.) [Incluye fragmentos de: El pozo ("Ana Maria"), Tierra de nadie ("Nené"), Para esta noche ("Victoria"), La vida breve ("Elena" y "La Queca"), Los adioses ("La muchacha de las sierras"), Una tumba sin nombre ("Rita"), El astillero ("Josefina-Angélica Inés" y "La mujer de Gálvez"), Juntacadáveres ("Las mujeres") y La cara de la desgracia ("La muchacha de la playa").]

* Tiempo de abrazar y los cuentos de 1933 a 1950. Montevideo: Arca Editorial S.R.L., 1974. 247 pp. (Precede: Jorge Ruffinelli, "Onetti antes de Onetti”, pp. XI-LIV). [Incluye: "Avenida de Mayo-Diagonal-Avenida de Mayo", "El obstáculo", "El posible Baldi", "Convalescencia", "Un sueño realizado", "Mascarada", "Bienvenido, Bob", "La larga historia", "Nueve de Julio", "Regreso al sur", "Esbjerg, en la costa", "La casa en la arena", "Los niños en el bosque" y Tiempo de abrazar. 1

\section{FRAGMENTOS DE NOVELAS}

“Excursión." Marcha (Montevideo), Año V, No. 176 (19 de marzo de 1943), p. 14. (Fragmento de Tiempo de abrazar.)

"Tiempo de abrazar." Capítulos XI y XIX. Marcha (Montevideo), Año VI, No. 189 (25 de junio de 1943), 2a sección, pp. 4-5. Capitulo XI reproducido en Crisis (Buenos Aires), No. 2 (junio de 1973), pp. 36-37. (Fragmento de Tiempo de abrazar.)

"Tiempo de abrazar." Marcha (Montevideo), Año VI, No. 216 (31 de diciembre de 1943), pp. 18-19. (Fragmento de Tiempo de abrazar.)

" "De La vida breve." El País (Montevideo), Año XLIV, No. 14.024 (28 de enero de 1962), p. 6. (Fragmento de La vida breve.)

" "Un fragmento de El astillero." El Pais (Montevideo), Año XLIV, No. 14.024 (28 de enero de 1962), p. 6. (Fragmento de El astillero.)

['Véanse todos los fragmentos de Las máscaras del amor en Colecciones de cuentos y novelas.] 
"“Tres textos de Onetti"', en Onetti, Jorge Ruffinelli, preparador. Montevideo: Biblioteca de Marcha, 1973, pp. 7-8. Incluye: "Autorretrato" (de La Vida breve) y dos fragmentos inéditos, "Infancia" y "Credo", que no son de novelas.

["Véanse "Los niños en el bosque" (fragmentos de un borrador de novela) y Tiempo de abrazar (que incluye todos los fragmentos asequibles de la novela) de Tiempo de abrazar y los cuentos de 1933 a 1950 en Colecciones de cuentos y novelas.]

\section{ARTICULOS PERIODISTICOS}

Los siguientes aparecen en Marcha (Montevideo) con la firma "Periquito el Aguador":

"La piedra en el charco." Año I, No. 6 (28 de julio de 1939), p. 2.

"La piedra en el charco." Año I, No. 7 (4 de agosto de 1939), p. 2.

“La piedra en el charco: Katherine y ellas." Año I, No. 9 (18 de agosto de 1939), p. 2.

“La piedra en el charco.” Año I, No 10 (25 de agosto de 1939), p. 2.

“La piedra en el charco." Año I, No. 11 (1 de septiembre de 1939), p. 2.

“La piedra en el charco.” Año I, No. 19 (27 de octubre de 1939), p. 2.

"Nueva edición de Sombras sobre la tierra." Año I, No. 23 (24 de noviembre de 1939), p. 3.

“La piedra en el charco." Año I, No. 26 (15 de diciembre de 1939), p. 3.

"La piedra en el charco: Propósitos de Año Nuevo." Año I, No. 28 (30 de diciembre de 1939), p. 4.

"La piedra en el charco: Regreso de la guerra locuaz." Año II, No. 75 (29 de noviembre de 1940), p. 5.

"La piedra en el charco: Mr. Philo Vance, detective." Año II, No. 76 (6 de diciembre de 1940), p. 23.

"La piedra en el charco: Un jueves literario." Año II, No. 77 (13 de diciembre de 1940), p. 22 
"La piedra en el charco: Jóvenes, se necesitan." Año II, No. 78 (20 de diciembre de 1940), p. 14.

“La piedra en el charco." Año III, No. 80 (10 de enero de 1941), p. 22.

“La piedra en el charco." Año III, No. 82 (31 de enero de 1941), p. 19.

Los siguientes aparecen en Marcha (Montevidec) con la firma "Grucho Marx":

"El corso a contramano: Se regala una idea." Año II, No. 75 (29 de noviembre de 1940), p. 5.

“El corso a contramano: Churchill-Marx." Año II, No. 76 (6 de diciembre de 1940), p. 5.

"El corso a contramano: Como me lo contaron." Año II, No. 77 (13 de diciembre de 1940), p. 5.

“Sin tema." Año II, No. 78 (20 de diciembre de 1940), p. 5.

“'La guerra permanente.” Año III, No. 80 (10 de enero de 1941), p. 5.

“Ruderico I de Borgoña." Año III, No. 82 (31 de enero de 1941), p. 5.

“'Autobrulote."' Año III, No. 84 (14 de febrero de 1941), p. 5.

“Estilo gráfico." AñoIII, No. 85 (21 de febrero de 1941), pp. 5 y 12.

“¡Ay de los tibios!”, Año III, No. 86 (28 de febrero de 1941), p. 5.

"Llamado al pais." Año III, No. 87 (7 de marzo de 1941), p. 5.

"Se llama Andresillo." Año III, No. 88 (14 de marzo de 1941), p. 5.

“'Dejad que los niños..." Año III, No. 89 (21 de marzo de 1941), p. 5.

“Salud al primer cruzado." Año III, No. 90 (28 de marzo de 1941), p. 5

“¿Xenofabias a mí?” Año III, No.91 (4 de abril de 1941), p. 5.

“Inútil para sordos." Año III, No. 93 (25 de abril de 1941), p. 5. 
" "De Juan Carlos Onetti." Acción (Montevideo), Año XIV, No. 4839 (29 de julio de 1962), p. 5.

\section{MISCELANEA}

Prólogos

"Prólogo"' en Arlt, Roberto. I sette pazzi. Milano: Bompiani Editores, 1971. Reproducido (con el título) "Arlt por Onetti: Semblanza de un genio rioplatense" en Marcha (Montevideo), Año XXXII, No. 1545 (28 de mayo de 1971), pp. 12-13. También reproducido (con el título) "Semblanza de un genio rioplatense" en Nueva novela hispanoamericana II. Jorge Lafforgue, compilador. Buenos Aires: Editorial Paidós, 1972, pp. 363-377.

"Prólogo" en Molina, Carlos Denis. Lloverá siempre. Montevideo: Arca Editorial, S.R.L., 1967, pp. 7-8.

Conferencia

"“Por culpa de Fantomas." Cuadernos Hispanoamericanos (Madrid), No. 284 (febrero de 1974), pp. 221-228.

\section{ENTREVISTAS, REPORTAJES Y ENCUESTAS}

*"Diálogo con Juan Carlos Onetti." La Cultura en México, Suplemento de Siempre (México), Vol. VI, No. 1053 (agosto de 1973).

"Encuesta entre escritores nacionales: 'la creación lleva implícita--se quiera o no--la denuncia...'." El Popular (Montevideo), Año V, No. 1742 (26 de enero de 1962), Suplemento de Cultura, p. 4.

*" "Juan Carlos Onetti: 'Un acto de amor'." Crisis (Buenos Aires), Año 1, No. 2 (junio de 1973), pp. 30-31.

*" "Opiniones sobre el decreto de censura." Marcha (Montevideo), Año XXXIII, No. 1576 (30 de diciembre de 1971). p. 31.

"Soriano, Osvaldo. "Reportaje al gran escritor uruguayo: Juan Carlos Onetti o la espera de la obra redonda." La Opinión Cultural(Buenos Aires), 29 de abril de 1973, pp. 8-9.

* "William Faulkner a través de hombres del Uruguay." Acción (Montevideo), Año XIV, No. 4826 (15 de julio de 1962), p. 5.

LIBROS, TESIS Y COLECCIONES DE ENSAYOS SOBRE ONETTI

${ }^{*}$ Deredita, John. Disintegration and Dream Patterns in the Fiction of Juan Carlos Onetti. Tesis doctoral inédita. Yale University, 1973. 305 pp.

* Kadir II, Djelal. The Aesthetics of Juan Carlos Onetti's Novel. Tesis doctoral inédita. The University of New Mexico, 1972. 195 pp.

* Ruffinelli, Jorge, preparador. Onetti. Montevideo: Biblioteca de Marcha, 1973. 293 pp. [Incluye: "Tres textos de Onetti". Jorge Ruffinelli, "Cronología". Mario Benedetti, "La aventura del hombre". Ruben Cotelo, "Cinco lecturas de Onetti". Jaime Concha, "Conciencia y subjetividad en El pozo". Jaime Concha, "Sobre Tierra de nadie". Jorge Ruffinelli, "La historia secreta de Para esta noche". Hugo J. 
Verani, "En torno a Los adioses". Wolfgang A. Luchting, "El lector como protagonista de la novela." John Deredita, "E1 lenguaje de la desintegración. Notas sobre El astillero". Emir Rodríguez Monegal, "Conversación con Onetti". Hugo J. Verani. "Contribución a la bibliografía de Onetti".]

*Verani, Hugo J. La obra narrativa de Juan Carlos Onetti: Estructura y significación. Tesis doctoral inédita. University of Wisconsin, 1973. $305 \mathrm{pp}$.

ESTUDIOS, NOTAS Y RESENAS

*Adams, Michael Ian. Alienation in Selected Works of Three Contemporary Latin American Authors. Tesis doctoral inédita. University of Texas at Austin, 1972, pp. 67-128.

Alsina Thevenet, Homero, et. al. "El pozo de Juan Carlos Onetti veinticinco años después." Marcha (Montevideo) Año XXVI, No. 1225 (21 de mayo de 1965), p. 18. [Los otros autores: Eduardo H. Galeano, Clara Silva, Alberto Orregioni, Carlos Maggi y Mario C. Fernández.]

Anónimo. "Cuentos completos por Juan Carlos Onetti."' La Nación (Buenos Aires), Año XCVIII, No. 34.493 (22 de octubre de 1967), 3a sección, p. 5.

*..... "Juan Carlos Onetti: La muerte y la niña." Marcha (Montevideo), Año XXXV, No. 1665 (21 de diciembre de 1973), p. 30.

*..... "Presencia de Onetti en Madrid." Mundo Hispánico (Madrid), Año XXVII, No. 310 (enero de 1974), pp. 54-55.

*.--.-. "A Leading Writer is Held in Uruguay." The New York Times (New York), Vol. CXXIII, No. 42,402 (February 26, 1974), p. 8.

*-..-. "Uruguay in Decline, Awaits Full Military Takeover."' The New York Times (New York), Vol. CXXIII, No. 42,417 (March 13, 1974), p. 2.

*-.... "El caso Onetti."' Cosmos (Xalapa, México), No. 7 (1974), pp. 1-7.

Bonnefoy, Claude. 'Une ville toute rouillée." Le Nouvel Observateur (Paris), Vol 18, No. 161 (13 décembre 1967), p. 38.

Bordelois, Yvonne A. "Juan Carlos Onetti: Tan triste como ella." Cuadernos (París), No. 98 (julio de 1965), pp. 85-86.

Bueno, Salvador. "Onetti, Juan Carlos. Juntacadáveres." Sin Nombre (San Juan, Puerto Rico), Vol. 2, No. 3 (enero-marzo de 1972), pp. 90-93. Reproducido con el título "Juniacadáveres, ¿novela social?" en Indice (Madrid), Año XXVI, No. 284285 (1-15 de febrero de 1971), pp. 61-62.

Carrillo, Bert Bono. The Alienated Hero in Four Contemporary Spanish American Novels. Tesis doctoral inédita. University of Arizona, 1970, pp. 1-6 y 48-81.

"Castillo, Guido. "Imagen de Juan Carlos Onetti." Mundo Hispánico (Madrid), Año XXVII, No. 310 (enero de 1974), p. 54.

Cotelo, Ruben. "Realidad y creación en la última novela de Onetti." El Páis (Montevideo), Año XLII, No. 13.330 (18 de octubre de 1959), p. 6.

-.--.. "El guardi ín de su hermano." El Pais(Montevideo), Año XLIII, No. 13.629 (12 de diciembre $d \geq 1960$ ), p. 28.

-. "Humor : desesperación en Juan Carlos Onetti."' El Pais(Montevideo), Año XLIV, No. 14.23 ( 27 de agosto de 1962), p. 12. 
-.-.-. "Muchacha y mujer." El Pais (Montevideo), AñoXLVI, No. 14.569 (16 de febrero de 1964), p. 7.

-.--. "Arquetipo de la pareja viril."' El Pais (Montevideo), Año XLVII, No. 15.489 (14 de agosto de 1966), p. 6.

*...-., preparador. "El invierno de nuestro descontento" en "Los contemporáneos". Capitulo Oriental 2: La bistoria de la literatura uruguaya. Montevideo: Centro Editor de América Latina, 1968, p. 25.

D.T.F. Nota que precede a "Mercado viejo" de Juan Carlos Onetti. Accion (Montevideo), No. 1606 (10 de diciembre de 196?), p. 8.

"Díez, Luis Alfonso. "Prefacio" en The Formal Expression of Meaning in Juan Carlos Onetti's Narrative Art de Yvonne Perier Jones. Cuernavaca: Centro Internacional de Documentación, 1971, pp. 3-7.

"E. del C. "Viñetas y anotaciones." Revista Mexicana de Cultura, Suplemento de El Nacional(México), 11 de marzo de 1973, p. 2.

Franco, Jean. La cultura moderna en América Latina. México: Editorial Joaquín Mortiz, S.A., 1971, pp. 209, 210, 228, 235, 245, 261 y 268.

"Frankenthaler, Marilyn. "On the Fate of a Novelist: To the Editor." The New York Times (New York), Vol.CXXIII, No. 42,416 (March 12, 1974), p. 36.

Gasca, Argelio. "Breves y harapientas notas sobre Juan Carlos Onetti." Diorama de la Cultura, suplemento de Excélsior (México), 23 de agosto de 1970, p. 14.

Gertel, Zunilda. "La novela personal existencial" è La novela bispanoamericana contemporanea. Buenos Aires: Editorial Columba, 1970, pp. 83-88.

Ghiano, Juan Carlos. "Juan Carlos Onetti y la novela." Ficción (Buenos Aires), No. 5 (enero-febrero de 1957), pp. 247-253.

"Gibbs, Beverly J. “Ambiguity in Onetti's El astillero." Hispania (Appleton, Wisconsin), Vol. 56, Special Issue (April, 1973), pp. 260-269.

*Goic, Cedomil. Historia de la novela hispanoamericana. Santiago de Chile: Ediciones Universitarias de Valparaíso, 1972, pp. 217-222 y 229-235.

"Grande, Félix. "Con Onetti." Plural. Revista mensual publicada por Excélsior (México), Vol. 1, No. 2 (noviembre de 1971), pp. 13-16.

"Gregorich, Luis. "Juan Carlos Onetti'" en Cuentos de dos orillas. B. Sarlo Sabejanes, seleccionador. Buenos Aires: Centro Editor de América Latina, 1971, p. 127.

"Jaramillo Levi, Enrique. “Ambigüedades temáticas en el cuento 'Bienvenido, Bob', de J. C. Onetti."' Nueva Narrativa Hispanoamericana (Garden City, New York), Vol. IV, No. doble (enero y septiembre de 1974), pp. 357-360.

"Krebs, Albin. "Notes on People." The New York Times (New York), Vol. CXXIII, No. 42,490 (May 25, 1974), p. 23.

"Levine, Suzanne Jill. "Last Battle for a Free Press in Uruguay." The New York Times Book Review (New York), Vol. CXXIII, No. 42,477 (May 12, 1974), p. 47.

López Ruiz, Juvenal. "Onetti, un novelista del sino." Imagen (Caracas), No. 35 (15-30 de octubre de 1968), p. 4.

"Manjárrez, Héctor. "Onetti: el infierno son los demás que soy yo mismo." La Cultura 
en México. Suplemento de Siempre (México), No. 538 (31 de mayo de 1972), pp. VIVIII.

"Montaner, Carlos Alberto. "Onetti, figura de las letras de Hispanoamérica." $A B C$ (Madrid-edición aérea), Año XXV, No. 1262 (7 de marzo de 1974), pp. 11-12.

"Paganini, Alberto, Alejandro Paternain y Gabriel Saad. 'Onetti, Juan Carlos' en Cien autores del Uruguay. Buenos Aires: Centro Editor de América Latina, 1969, pp. 5658.

Pollmann, Leo. La "nueva novela" en Francia y en Iberoamérica. Traducción de Julio Linares. Madrid: Editorial Gredos, S.A., 1971, pp. 83-89, 133-139 y 228.

* Rama, Angel. La generacion critica: 1939-1969. 1. Panoramas. Montevideo: Arca Editorial, S.R.L., 1972, pp. 33-39, 93 y 120-129.

"Raviolo, Heber. 'Onetti premiado en Italia: La vuelta del narrador." Marcha (Montevideo), Año XXXVI, No. 1674 (8 de noviembre de 1974), p. 32.

"Rodríguez Monegal, Emir. "Situación del cuento uruguayo en 1956." Marcba (Montevideo), Año XVIII, No. 845 (28 de diciembre de 1956), 2a sección, pp. 26-28.

-.-.-. "Una forma del fragmentarismo." El Pais (Montevideo), Año XLVII, No. 15.060 (16 de mayo de 1965), p. 21.

*-.-. " "The New Novelists." Encounter(London), Vol. XXV, No. 3 (September, 1965), pp. 98-109.

*----. "Presentación" en Las máscaras del amor de Juan Carlos Onetti. Buenos Aires: Centro Editor de América Latina, 1968, p. 7.

*-.-- "On the Fate of a Norelist: To the Editor." The New York Times (New York), Vol. CXXIII, No. 42, 416 (March 12, 1974), p. 36.

*...-. and Seventeen Others. "Suppression in Uruguay: To the Editors." The New York Review of Books (New York). Vol. XXI, No. 5 (April 4, 1974), p. 23

"Ruffinelli, Jorge. "La ocultación de la historia en Para esta noche de Juan Carlos Onetti.", Nuevos Aires (Buenos Aires), Año 3, No. 9 (diciembre de 1972-enero-febrero de 1973), pp. 23-38. Reproducido en Nueva Narrativa Hispanoamericana (Garden City, New York), Vol. III, No. 2 (septiembre de 1973), pp. 145-159.

*-..... "De la violencia y otras enajenaciones." Marcha (Montevideo), Año XXXIV, No. 1639 (13 de abril de 1973), p. 29.

*-..-. "Onetti al cine." Marcha (Montevideo), Año XXXIV, No. 1647 (15 de junio de 1973), p. 29. (Reportaje a Julio Jaimes.)

*...-. "'Onetti antes de Onetti”' en Tiempo de abrazar y los cuentos de 1933 a 1:50. Montevideo: Arca Editorial S.R.L., 1974, pp. XI-LIV.

"Schoijet, Mauricio. "Suppression in Uruguay: To the Editors." The New York Review of Books (New York), Vol. XXI, No. 5 (April 4, 1974), p. 23.

*Schwartz, Kessel. "Themes, Trends and Textures: The 1960's and the Spanish American Novel." Hisfania (Appleton, Wisconsin), Vol. 55, No. 4 (December, 1972), pp. 817-831.

Sorel, Andrés. "La nueva novela latinoamericana, I: U1uguay, Bolivia, Chile." Cuadernos HIspanoamericanos (Madrid), No. 191 (noviembre de 1965), pp. 221-238. 
"T.J.D. Nota que precede a "El entierro del chivo" de Juan Carlos Onetti. Marcha (Montevideo), Año XXI, No. 977 (8 de septiembre de 1959), 2a sección, pp. 6 y 16.

"Umbral, Francisco. "Juan Carlos Onetti." Ya (Madrid), Año XXXVI, No. 9.954 (13 de junio de 1970), p. 85.

Vanasco, Alberto. "Juan C.arlos Onetti, Los adioses." Histonium (Buenos Aires), Año 16, No. 188 (enero de 1955), p. 61.

*---.. “Juan Carlos Onetti y la creación literaria." Ponencia leída en el XVI Congreso del Instituto Internacional de Literatura Iberoamericana, Michigan State University (East Lansing, Michigan), 26-31 de agosto de 1973, pp. 1-6.

$\mathrm{V}$ [isca], A [rturo] IS [ergioI. Nota que precede a "Naturaleza muerta" de Juan Carlos Onetti. Asir(Mercedes, Uruguay), No. 21 (abril de 1951), pp. 41-42.

-.--.. "Desarraigo." El Pats (Montevideo), Año XLVI, No. 14.445 (29 de septiembre de 1963), p. 6.

-.-..."Onetti, Jacob y el otro." El pats (Montevideo), Año L, No. 15.925 (3 de diciembre de 1967), p. 2 y Año L, No. 15.932 (10 de diciembre de 1967), p. 2.

*......'Juan Carlos Onetti"' en Alborada cuentos de hoy.Montevideo: Editorial Cisplatina, s.f., pp. 49-50.

Yankas, Lautaro. "Uruguay" en "Valores de la narrativa hispanoamericana actual." Cuadernos Hispanoamericanos (Madrid), No. 236 (agosto de 1969), pp. 344-347. 
